www. revistad y o. com

\title{
Experiencia docente semivirtual de la asignatura Dirección Estratégica Empresarial durante el confinamiento por la covid-19, desde un enfoque de sistemas dinámicos
}

Justo de Jorge Moreno

Recibido: 7 de Enero de 2021

https://doi.org/10.37610/dyo.v0i75.611

Aceptado: 12 de Abril de 2021

\section{Resumen}

Este trabajo, se ha planteado como objetivo documentar el proceso de enseñanza-aprendizaje, en la asignatura de Dirección Estratégica Empresarial de $4^{\circ} \mathrm{ADE}$ en el curso 2020, a partir de la elaboración de un trabajo de investigación, por medio de un proceso pedagógico, donde el aprendizaje es construido, autónomo y colaborativo. Para ello se ha utilizado la metodología de sistemas dinámicos. A partir de la hipótesis dinámica y por tanto del establecimiento de las relaciones causales, que asocian motivación (desde sus diferentes perspectivas) de los alumnos/grupos y del docente con el proceso de aprendizaje. Los principales resultados alcanzados, por medio de la simulación y de los escenarios planteados han sido los siguientes; i) el modelo planteado refleja el comportamiento del proceso de enseñanza y su validación se ajusta razonablemente a la información y percepción del proceso y ii) las suposiciones en condiciones extremas proporcionaron respuestas relativamente razonables.

\section{Palabras clave}

Proceso enseñanza-aprendizaje, sistemas dinámicos, motivación, Trabajo de investigación

\section{Introducción}

La implantación del Espacio Europeo de Educación Superior ha traído consigo una creciente preocupación por un nuevo modelo de enseñanza-aprendizaje, centrado en el alumno. Conceptos como "competencias, habilidades o destrezas" emergen de forma relevante y como criterios clave en el nuevo planteamiento de la Enseñanza Superior. El énfasis en la evaluación del rendimiento, en lugar del proceso de aprendizaje del alumno, parece ser la tendencia dominante en el ámbito universitario y en la enseñanza en general. Los problemas derivados de este tipo de evaluación del rendimiento, asociados a la nota de los exámenes, es probable que se relacionen con el aprendizaje, a corto plazo y con una importante motivación extrínseca "estudio porque tengo un examen". Por tanto, quizás no se vincule excesivamente, por la curiosidad o la necesidad de adquirir nuevos conocimientos que guíen a los alumnos hacia un aprendizaje significativo. Es decir, el conocimiento transmitido pierde relevancia a favor del conocimiento construido, autónomo y colaborativo, y por lo tanto la motivación intrínseca y de logro juega un papel relevante.

Justo de Jorge Moreno *

justo.dejorge@uah.es

iD

https://orcid.org/0000-0002-8326-3046

* Dpto. Economía y Dirección de Empresas Facultad de Ciencias Económicas, Empresariales y Turismo. Plaza de la Victoria, 2, Alcalá de Henares, Madrid, España.Universidad de Alcalá.
Sin embargo, no será cosa fácil la transición desde el conocimiento transmitido al construido, la propuesta de cuestiones novedosas y elaboración de significados originales que se situé a un nivel superior, implica la incursión en actividades de mayor nivel de exigencia, con tendencia divergente y asunción de incertidumbre (diversas respuestas posibles y carácter heterogéneo). Finkel (2008:42) define la $<<$ buena docencia $>>$, como crear aquellas circunstancias que conducen al aprendizaje relevante en terceras personas. Subraya que el concepto aprendizaje tiene especial relevancia. Algunos de sus planteamientos se relacionan con que aprender es el objetivo y enseñar es el medio. Llama la atención cuando indica que, los docentes no deberían olvidar ese objetivo, cuando idean formas de dar clase. Los alumnos, por su parte, emergen en el proceso de aprendizaje como implicados directos en su propio proceso de formación. De acuerdo con Ferry (1997), todo proceso de formación implica $<<$ formarse para adquirir cierta forma $>>$, por lo que enseñanza y aprendizaje son soportes de la formación; pero la formación implica, además, desarrollo personal.

Este trabajo, tiene como objetivo documentar el proceso de enseñanza-aprendizaje en la asignatura de Dirección Estratégica Empresarial de $4^{\circ} \mathrm{ADE}$ (en adelante DEE), a partir de la elaboración de un trabajo de investigación, por medio de un proceso pedagógico, donde el aprendizaje es construido, autónomo y colaborativo. La importancia de los conceptos de la asignatura en la gestión de empresas es relevante, pero el énfasis de esta se relaciona con ayudar a los alumnos a comprender mejor los problemas inherentes a la toma de decisiones en la empresa, dejando un amplio margen de maniobra a la hora de elegir el tema del trabajo. Esto implica, por ejemplo, no restringir ni el tipo de organización a estudiar según titularidad, pública o privada, el ámbito temporal o espacial, entre otros. 
Tampoco, se imponen criterios restrictivos sobre vinculaciones de la gestión de empresas con otras materias, teoría de juegos, investigación operativa, economía con mayor o menor enfoque social, etc. En el anexo I se ofrece un breve resumen de algunos de los trabajos realizados. Finalmente, la apuesta por un contenido eminentemente práctico es crucial, ya sea cualitativo o cuantitativo o una combinación de ambos. Parte de la literatura relativa a la enseñanza, como por ejemplo Getachew (2018) reconoce que la retención de conocimientos de forma cuantitativa, basada en la investigación mejora el proceso de aprendizaje, en comparación, con la lectura y aprendizaje memorístico (Kwon et al., 2005).

Para llevar a cabo este estudio, el trabajo se estructura de la forma siguiente. En la sección 2 se muestran la metodología y modelos. Esta sección se estructura en diversas subsecciones, desde los arquetipos sistémicos hasta el modelo global. Así mismo, se muestran los diferentes resultados de los modelos y su simulación. La sección 3, muestra la evaluación de los alumnos sobre el proceso de enseñanza aprendizaje. Finalmente, la sección 4, muestra las principales conclusiones, recomendaciones y extensiones, para futuros trabajos.

\section{Metodología y modelos}

\subsection{Metodología}

En esta sección se documenta el proceso de enseñanzaaprendizaje de DEE. Para ello se utiliza el método de desarrollo de sistemas dinámicos (SD) a través de la utilización del software de simulación Vensim PLE. De acuerdo con Forrester (1968) la dinámica de sistemas es una metodología para comprender el cambio o transformación, utilizando para ello, ecuaciones diferenciales. Como menciona PerezAnsede (2015), en análisis complejos en las que es necesario trabajar con un número incierto de parámetros difíciles de cuantificar, según Martin (2003), se pueden utilizar modelos menos formales que los normalmente empleados cuando los estudios abordan enfrentarse a una realidad con parámetros limitados y cuantificables. La utilización de SD permite una propuesta de mayor nivel estructural del objetivo a investigar y de posibles soluciones.

En los SD se identifican diferentes tipos de variables; de nivel, relacionadas con aquellas que acumulan magnitudes en el tiempo y que representan el establecimiento del modelo propuesto, la dinámica inherente o el paso del tiempo. Su formulación sería, la que muestra la ecuación 1:

Las variables nivel, están influenciadas por otras variables que se acumulan en ellas, denominadas variables flujo, cuya misión es provocar cambios en las variables nivel. Adicionalmente, se encuentran las variables auxiliares, que reciben y proporcionan información al modelo, por medio de una función definida influyendo al resto de variables mencionadas (nivel, flujo y auxiliares). No obstante, como menciona Perez-Ansede (2015) las variables auxiliares pueden ser empleadas para facilitar una mayor comprensión del modelo propuesto (Weber, 2010).

Finalmente, los parámetros, o constantes, tienen valores que están determinados de forma exógena, permitiendo llevar a cabo interesantes análisis de sensibilidad del modelo, y experimentar cambios en el mismo.

La utilización de SD implica tener en cuenta las siguientes consideraciones. Como menciona Sukhwal (2015), en los $\mathrm{SD}$ se identifican tres importantes elementos; relaciones causa-efecto, bucles y retardos de tiempo. En el primer caso, los SD permiten combinar diferentes causas y efectos, lo que supone dar un paso más allá de los análisis de sistemas o relaciones aisladas, más fáciles de comprender, pero más alejadas del modelo real. La retroalimentación o bucle es el proceso donde un parámetro se ve influenciado por sí mismo y otros parámetros en un futuro cercano.

\subsection{Modelización del proceso}

Para la realización de los modelos se siguieron los planteamientos de Forrester (1968), Barlas (1996), Sterman (2000) y Homer (2019). Se llevo a cabo, una modelización incremental por fases, recogiendo el planteamiento general (conceptualización, formalización y evaluación, validación). Fase 1, creación de un modelo base preliminar, estableciendo las relaciones entre la elaboración del trabajo y las interacciones entre alumnos y estudiantes/grupos. En esta fase se recopila la información, se identifican las variables a considerar que permiten determinar los límites del sistema y la hipótesis dinámica del mismo. Se ha reflexionado, sobre las estructuras de realimentación (de refuerzo y equilibrio). En la fase 2, se ha abordado, la expansión del modelo añadiendo más parámetros y variables intentando capturar el proceso enseñanza-aprendizaje-investigación. En la fase 3, se ha validado el modelo por medio de la simulación.

Con el objetivo de una mayor comprensión del proceso, se explica brevemente la organización de la clase/grupos (En el anexo II, se ofrece un mayor nivel de detalle). La asignatura DEE en el curso 2020, estuvo integrada por 20 grupos con un total de 61 alumnos. La formación de los grupos fue voluntaria (2 a 5 alumnos) establecidos desde el inicio del curso. Debido a la especial circunstancia de la pandemia covid-19, se pusieron a disposición de los alumnos recursos adicionales a los utilizados en años anteriores. 


\subsubsection{Arquetipos sistemicos}

Quizás uno de los problemas inherentes al proceso enseñanza-aprendizaje impulsado por el proceso Bolonia, se relacione con la limitación de recursos con los que acometer las actividades necesarias, encaminadas a fomentar el autoaprendizaje del alumno. Esto podría asociarse con la necesidad de disponer, no solamente de recursos físicos, sino sobre todo de docentes suficientes y con la preparación y disposición, para asumir el reto. Todo ello teniendo en cuenta la situación actual de la covid-19, que agrava aún más el proceso.

En la figura 1, se muestra el arquetipo de "Erosión de metas", donde pueden verse dos bucles; "Ajustes de objetivo-Trabajos objetivo" y "Acciones de mejora-Estado actual" que se equilibran por medio de la brecha.
Figura 1 Arquetipo

"Erosión de metas".

Fuente: Elaboración propia

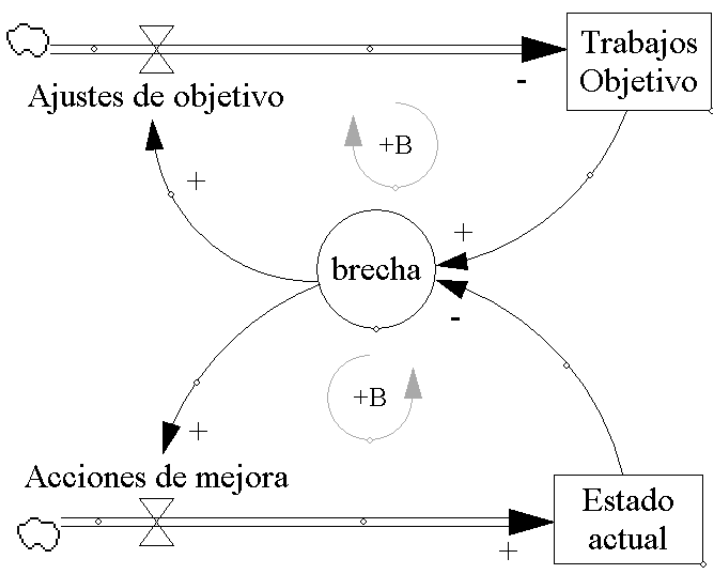

propuesta en proyectos de $\mathrm{I}+\mathrm{D}+\mathrm{i}$. Esta interpretación implica que el sistema mide su eficacia, en términos del, porcentaje de trabajos que alcanzan un nivel adecuado para su posterior desarrollo sobre el total de trabajos en marcha.
Figura 2 Comportamiento de las variables de salida de "Erosión de metas" (simulación con mejoras del $10 \%$ parte superior y con el $10 \%, 20 \%$ y $40 \%$ parte inferior).

Fuente: Elaboración propia
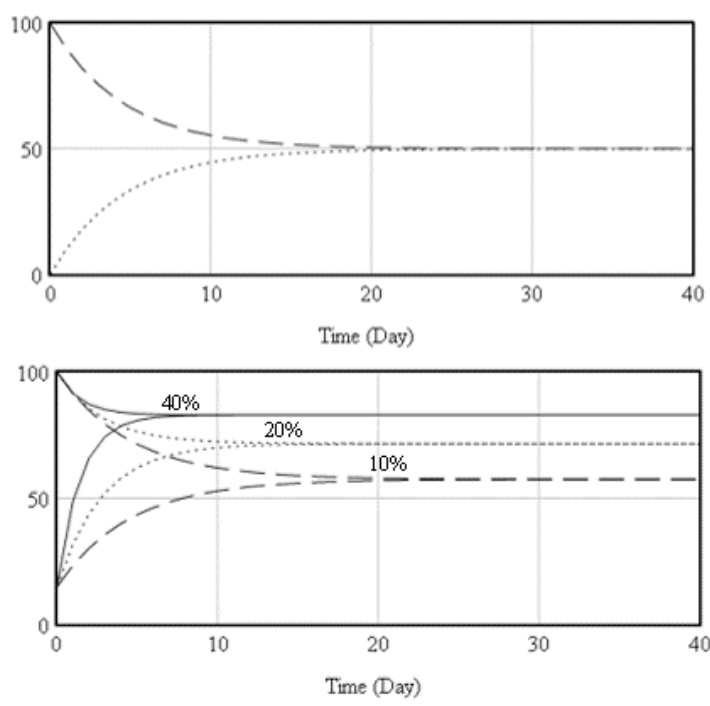
En la figura 2, parte superior, el comportamiento de los trabajos objetivo decrece para reducir la brecha, y del Estado actual, que alcanzan la meta, con incrementos de mejora de los grupos del 10\%, en la mediana del desarrollo de la asignatura, sobre la $20^{\mathrm{a}}$ clase.

En la parte inferior, se muestra la simulación considerando tambien incrementos del $20 \%$ y $40 \%$. Como puede apreciarse, la absorción de mejoras según grupos identifica diferencias relevantes de los puntos de convergencia (donde se encuentran los valores porcentuales situados). En el caso, del escenario con niveles de mejora del $40 \%$, sería la $8^{\text {a }}$ clase, lo que puede suponer gradientes relevantes de calidad final del trabajo. Se abordará este tema con mayor detalle en el proceso de simulación del modelo global.

Entre las restricciones que puedan dificultar alcanzar las mejoras de rendimiento en la elaboración del trabajo, se encuentran; la tutorización del proceso enseñanzaaprendizaje, por la existencia de excesivos trabajos y una heterogeneidad relevante en los conocimientos de los grupos/estudiantes, habilidad y motivación, que, en algún caso, podrían retrasar la finalización de los trabajos o incluso colapsar el proceso. Desde el punto de vista de la literatura, cuando se analiza este modelo, se alude, a la presencia de cuellos de botella en el sistema, que puede describirse como la metáfora del "valle de la muerte".

\subsubsection{Modelo control de avances}

En esta subsección se aborda la simulación de un proceso de avance del trabajo con resolución de dudas. No se distingue entre tutoría asistencial e integral. La primera sería la que se refiere a aquellos grupos que se bloquean con cierta frecuencia y tienen un alto grado de dependencia del profesor. Quizás por la dificultad que encuentran en la realización del trabajo de complejidad media en relación con sus conocimientos o por un alto grado de compromiso, que les conduce a una elevada exigencia de profundización en el trabajo de calidad, por encima de la media. En el primer grupo, se encontrarían grupos/alumnos que, a pesar de haber asistido a explicaciones presenciales o virtuales de metodologías o conceptos determinados, la actividad les intimida. Por ejemplo, aplicación de modelos sencillos de econometría, la aplicación de técnicas no paramétricos de eficiencia o la utilización de planteamientos relativamente sencillos de teoría de juegos.

En el segundo, se encontrarían los grupos que empujan la frontera del nivel de aprendizaje, que podría considerarse medio o medio alto. Por ejemplo, investigar acerca de las estimaciones de audiencia televisiva a través de series temporales y su relación con los consejos de gobierno de las cadenas de televisión, con modelos de intervención o la utilización de tratamiento de modelos de regresiones de poisson, que asocien gestión estratégica del gasto público y victimas de género.

La información disponible, para él análisis, se relaciona con el registro de datos sobre las dudas significativas planteadas en diferentes grupos durante el periodo de tiempo de las clases. Concretamente, 4 grupos el $9^{\circ}$ día; 2 grupos el $13^{\circ}$ y 5 grupos el $22^{\circ}$. El supuesto que se adopta es que la relación entre las dudas planteadas y sus soluciones se simularan en el modelo, sobre el principio de que la asimilación tarda en el caso más desfavorable, siete días (menor tiempo afecta a menores transiciones mostradas en la figura 4), es decir de una semana a otra. La estructura del modelo en el software Vensim PLE utilizado, puede tener diferentes niveles de complejidad y soluciones, lo relevante, tal y como menciona la literatura mencionada, es que se reproduzca el proceso a través de la información disponible.

La figura 3, muestra el modelo con una variable estado, dos variables flujo y dos auxiliares. La variable estado "Grupos retrasados", captura la información instantánea del número de grupos en relación con el total. El flujo "Grupos nuevos retrasados", muestra la aparición de nuevos grupos en clase o fuera de ella. Mientras que "Grupos evolucionados", se refiere a aquellos que han solucionado las dudas. Con relación a las variables auxiliares, la relativa a la "Duración del proceso", se asocia a una constante, que como fue mencionado, se fuerza al caso más desfavorable de siete días.

La variable "Unidad de tiempo" simplemente se muestra para dar coherencia de unidades al modelo. Finalmente, la nube o sumidero, que genera de forma automática el entorno de simulación como origen del flujo de "Grupos nuevos retrasados", en la parte izquierda del modelo, mientras que, en la parte derecha, "Grupos evolucionados". 
Figura 3 Modelo avance de grupos.

Fuente: Elaboración propia

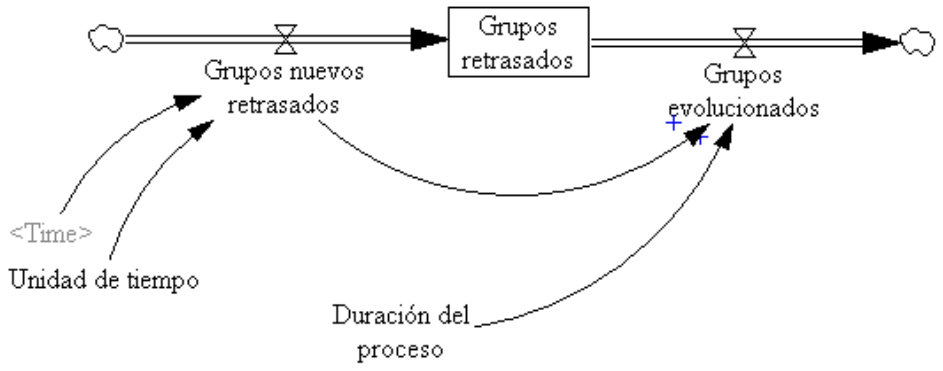

La simulación que aparece en la figura 4, muestra las dos variables de flujo "Grupos nuevos retrasados y evolucionados". Los "Grupos nuevos retrasados" aparecen con valores de 4, 4 y 5 los días 9, 13 y 22 del periodo de clases, gráficos de la parte superior izquierda.
Figura 4 Modelo avance de grupos

Fuente: Elaboración propia

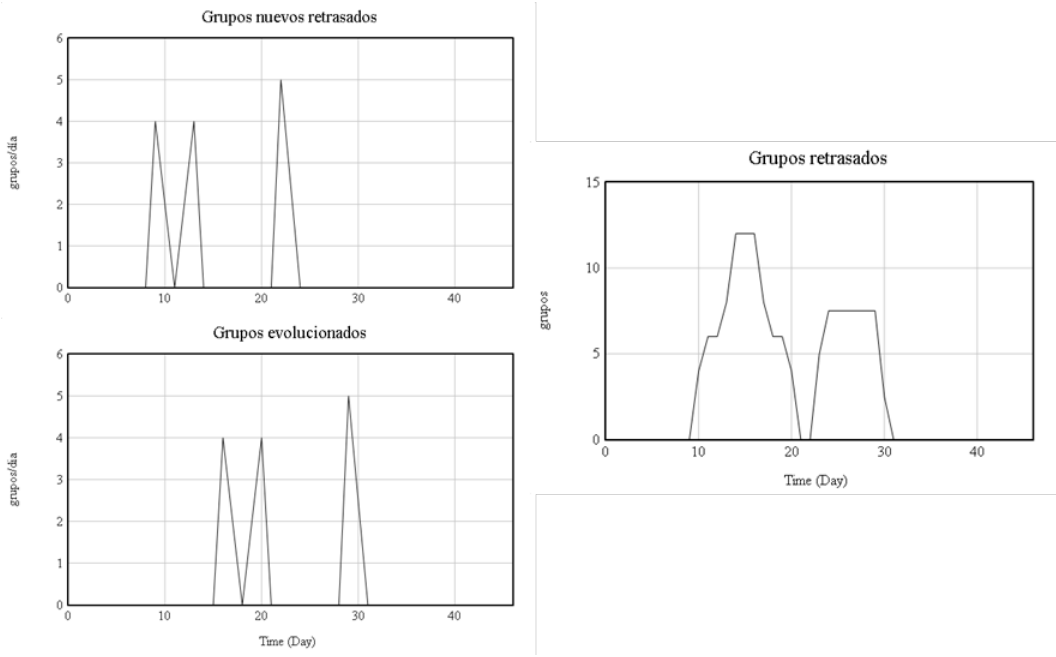

En la parte inferior izquierda se verifica la solución de dudas 7 días después en todos los casos. El número de "Grupos retrasados" en la clase evoluciona de forma escalonada con saltos ascendentes y descendentes con el desfase ya mencionado, parte derecha de la figura.

\subsubsection{Modelo global}

En esta sección se aborda el modelo global que pretende explicar el proceso de enseñanza-aprendizaje-investigación de DEE. De acuerdo con Robbins (2004) la propuesta se apoya en el campo de estudio del comportamiento organizacional. Se investiga el impacto que alumnos, grupos y estructuras organizativas tienen en la conducta dentro de las instituciones, en este caso la Universidad y en la posibilidad de mejora de la eficacia del sistema. De forma específica, se plantea un enfoque sistémico, que permita estudiar las relaciones de las partes del sistema pedagógico abordado. Se pretende atribuir causas y efectos soportados por procesos de simulación, bajo condiciones controlables a partir de datos obtenidos en la experiencia del curso 2020 y su posterior interpretación de manera razonable y rigurosa. Dentro del amplio campo de teorías de la motivación, generalmente clasificadas en; de contenidos (Maslow, Herzberg, McClelland, etc.) y asociadas con el entendimiento del individuo a través de sus necesidades y su vinculación con la motivación por medio de variables cognitivas o afectivas; o de procesos (Vroom, Equidad, Finalidad, etc.) asociadas con la forma en la que la situación del desarrollo del trabajo tiene lugar, que en su conjunto, explican con detalle el comportamiento de los alumnos en la Universidad y frente a la tarea a desarrollar.

A partir de la teoría de la Equidad basada en el concepto de disonancia cognoscitiva y la idea de comparación social, que explica como los individuos evalúan su opinión y deseos por medio de la comparación de ellos mismos con los demás. Esto se relacionaría con los procesos de evaluación global realizado en la asignatura, y las evaluaciones intra e intergrupos. La teoría de la finalidad permite reflexionar acerca de cómo el establecimiento de metas específicas genera mayores niveles de rendimiento que el establecimiento de objetivos generales y que las metas que son difíciles de alcanzar están directamente relacionadas con el rendimiento. Por último, la teoría de las expectativas de Vroom, relacionada con que las personas se sentirán motivadas a desarrollar ciertas 
actividades para alcanzar una meta si creen en el valor de esta y siente que sus acciones contribuirán a lograrla en cierto sentido. De forma más detallada y simple, el desempeño " $D$ " de los alumnos, es considerado como una función ( $\mathrm{f}$ ) de la interacción de la capacidad "C", (en términos de inteligencia y habilidades), con la oportunidad de desempeñarse " $O$ " y con la motivación "M".

De forma global, expresado como, $\mathrm{D}=\mathrm{f}(\mathrm{M}, \mathrm{C}, \mathrm{O})$. Ahora bien, el concepto de motivación se compone de dos tipos de esta, motivación extrínseca (ME) e intrínseca (MI). Recientemente Locke y Schattke (2019), mencionan que el debate sobre la motivación intrínseca y extrínseca ha ignorado casi por completo un tercer tipo crucial, a saber, la motivación de logro (ML), que a menudo se ha confundido con la motivación intrínseca. Los conceptos también han sufrido problemas de definición. Sugieren que los tres tipos de motivación son conceptos independientes pero relacionados (todos son fuentes de placer) que pueden facilitarse, compensarse o entrar en conflicto entre sí.
En la figura 5 se muestra el modelo global y por tanto la hipótesis dinámica que recoge el proceso de enseñanzaaprendizaje. En la parte superior de la figura aparecen tres variables de nivel que forma un bucle de crecimiento (color azul grueso). El modelo, podrían entenderse, por ejemplo, desde la variable "Motivación Global de los Grupos" (MGG), la cual se ve influenciada por la composición de las "Motivaciones, Intrínsecas, Extrínsecas, Necesidad de Logro" MI, ME, ML, respectivamente. Es relevante, considerar tambien el "Ruido los factores exógenos" (RFE) que pueden afectar a estas (color gris y rojo). donde cabe mencionar los derivados del confinamiento, como consecuencia de la covid-19, hacía el "Nivel de aprendizaje" (NAG). En concreto, la adquisición de conocimientos y habilidades, quien a su vez influye en el "Nivel de Investigación de los Grupos" (NIG), se trata de aplicar aquellos en el trabajo a realizar en función del objetivo de este y la información y metodologías necesarias para ello. Así mismo, incrementos en los NIG afectan a la MGG.
Figura 5 Modelo global Fuente: Elaboración propia

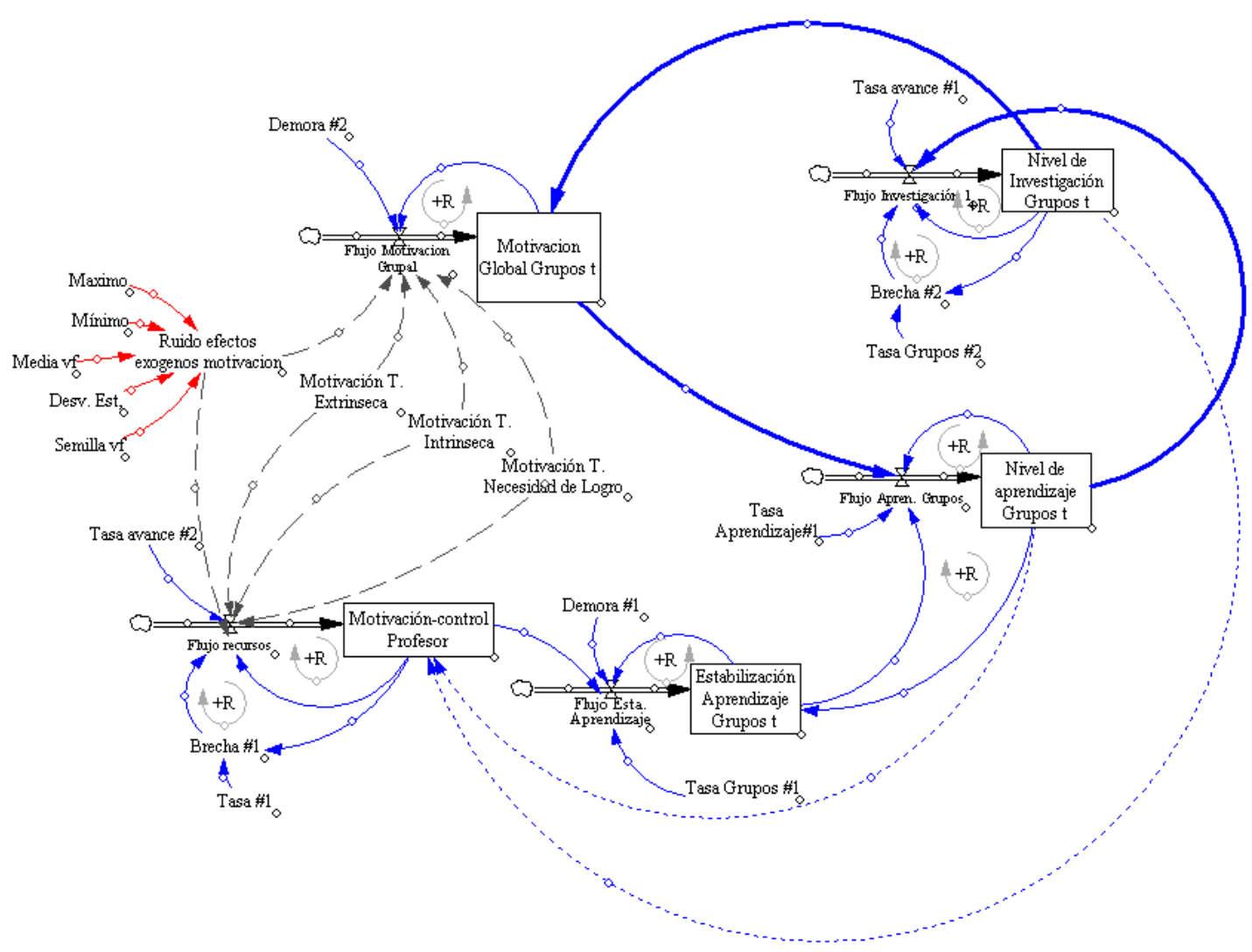


El NAG se relaciona con la necesidad de encontrar la "Estabilización de aprendizaje de los grupos" (EAG) necesaria del proceso enseñanza-aprendizaje de estos. Ya fue comentado anteriormente en los modelos de erosión de metas y de avances, la complejidad referida, a la diferencia de niveles de conocimientos y motivación intergrupo, así como al exceso del número de grupos existentes. Esta circunstancia la recoge EAG. A su vez la "Motivación y Control del Proceso del profesor" (MCP) contribuye de forma determinante sobre EAG. Finalmente, la MCP se ve influenciada al igual que en el caso de MGG tanto por MI, ME, ML como por RFE, y por NAG y NIG (color azul discontinuo).

\section{Proceso de simulación del modelo global}

En el ámbito de SD es habitual plantearse como objetivos que los comportamientos de las variables sigan patrones en las direcciones esperadas, en forma de evolución positiva o negativa, condiciones de estabilidad, etc. Para ello, los SD precisan de sistemas de control, que se caracterizan por la posibilidad de alterar parámetros que regulan el comportamiento de las variables deseadas. En el anexo 1, se encuentran la declaración de las variables.

La figura 6, muestra el comportamiento simulado de RFE y las MI, ME, ML que por su propia construcción tiene carácter aleatorio.
Figura 6 Variables exógenas Ruido, y Motivaciones Extrínseco, Intrínseco y de Necesidad de Logros Fuente: Elaboración propia

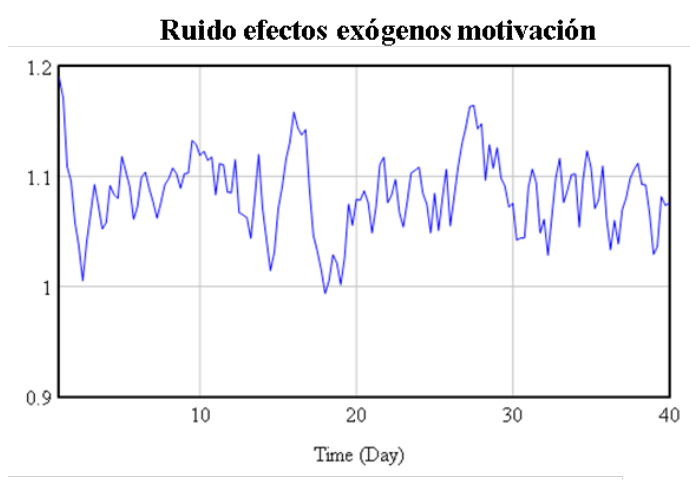

Tendencias de la Motivación

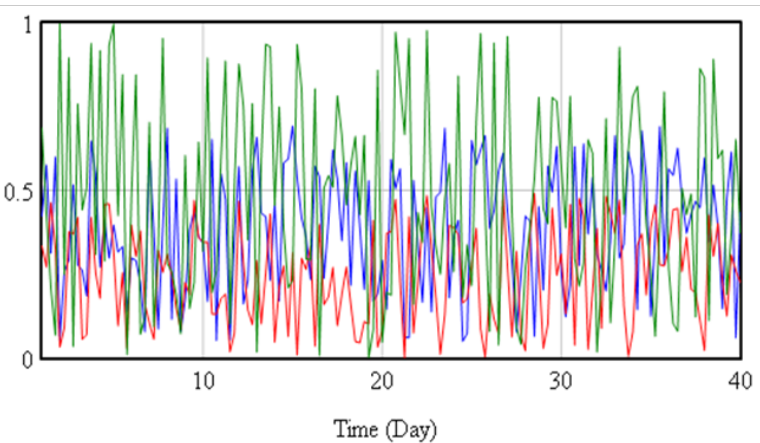

Time (Day)

— "Motivación T. Extrinseca" : Curren "Motivación T. Necesidad de Logro" : Current

Las actuaciones de simulación sobre Tasa de avance \#2, tienen un impacto relevante sobre el comportamiento del modelo. La figura 7, parte izquierda, muestra como avances o evoluciones en los trabajos según escenarios del 10\%,20\% y 30\% impactan significativamente en MCP. Se ha supuesto que estos avances tienen lugar en el $25 \%$ de los trabajos según el valor asignado a Tasa \#1. En la parte derecha de la figura 7, se muestra el dinamismo del flujo de recursos.
Figura 7 Simulación de Avances de los trabajos en el nivel y flujo de Motivación -Control del Profesor

Fuente: Elaboración propia
"Motivación-control Profesor"

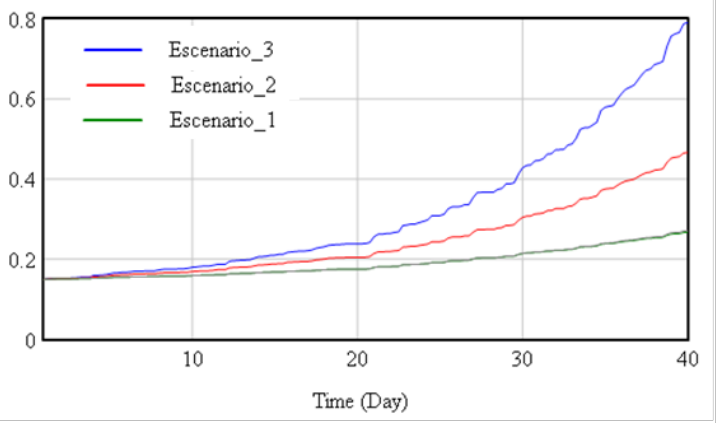

Flujo recursos

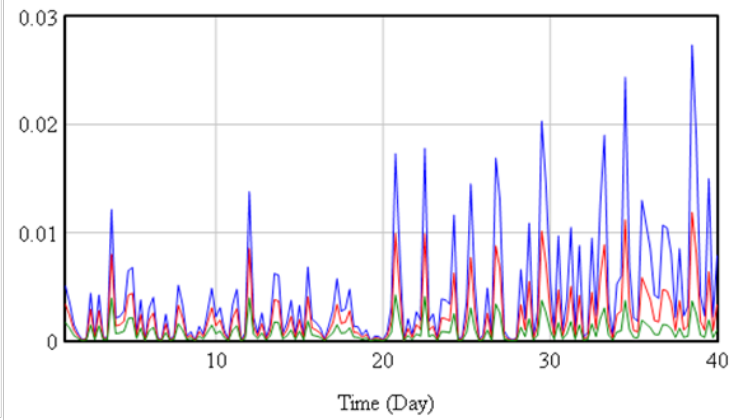


La influencia de Tasa de avance \#2 sobre EAG, como muestra la figura 8, parte izquierda supone que el proceso de enseñanza-aprendizaje es estable entre la $18^{\mathrm{a}}$ y $21^{\mathrm{a}}$ clase. Lo que supone aproximadamente cuatro clases después de finalizar, la serie de clases magistrales impartidas sobre conceptos en DEE (estrategia, gobierno de empresas, posicionamiento competitivo, etc) y algunas de las metodologías que contempla la asignatura de tipo cualitativo/ cuantitativo, como SISTRAT, Análisis Multicriterio con Excel, Estadística aplicada con Statgraphics o gretl y la introducción al análisis envolvente de datos (DEA por sus siglas en inglés).
El supuesto realizado en EAG, implica que la estabilización en el proceso de aprendizaje está condicionada por una demora o retardo, denominado "Demora \#1" media estimada para todos los grupos de una semana (el caso más complejo es el relativo a conceptos explicados, que se acompañan de la utilización de software). Si el tiempo de demora aumenta por término medio en un $20 \%$, como ilustra la parte derecha de la figura 8, la estabilización del proceso se vuelve complicada, y su inestabilidad, en términos de la diferencia de la asimilación de conceptos o metodologías entre grupos es difícil de gestionar.
Figura 8 Simulación de Avances en la Estabilización del Aprendizaje Fuente: Elaboración propia
Estabilización Aprendizaje en función de escenarios de avance

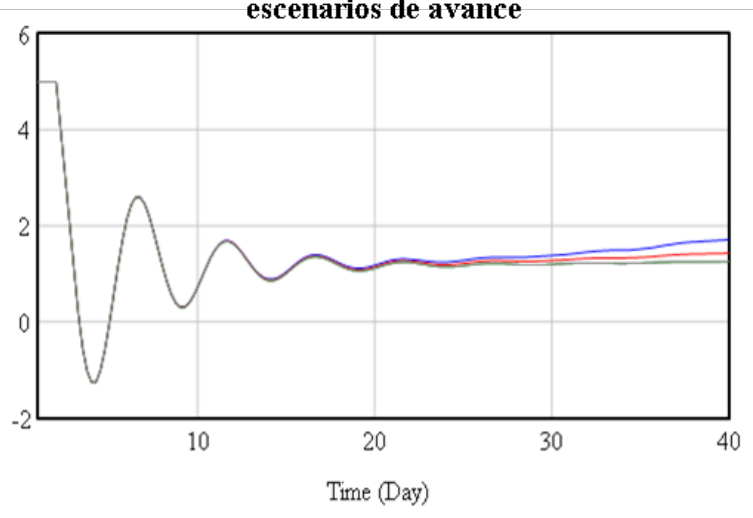

Inestabilidad del Aprendizaje en función de incrementos de demora

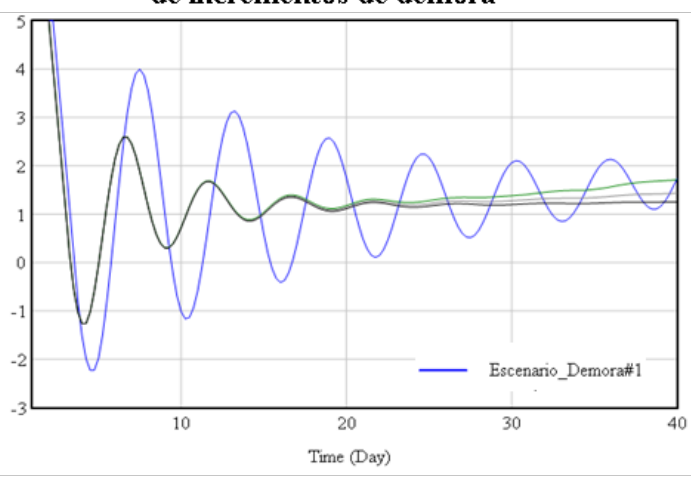

Los escenarios generados desde "Tasa de avance \#2", tiene su impacto en NAG, con una forma exponencial tal y como recoge la figura 9, parte superior izquierda. El cambio acusado de pendiente se refleja a partir del $70 \%$ aproximadamente de las clases (tutorías asistenciales e integrales). A raíz de este punto las diferencias de nivel de calidad de los trabajos se ponen claramente de manifiesto. Los gráficos de la parte superior derecha e inferiores, NAG, EAG y MCP muestra una simulación con avance del 50\% (asumiendo importantes niveles de heterogeneidad intergrupos) en la "Tasa de avance \#2". Como puede apreciarse, la trayectoria exponencial de NAG es ahora más acusada.
Figura 9 Simulación de

Nivel de Aprendizaje (3 y 4 escenarios)

Fuente: Elaboración propia
Nivel de aprendizaje Grupos t

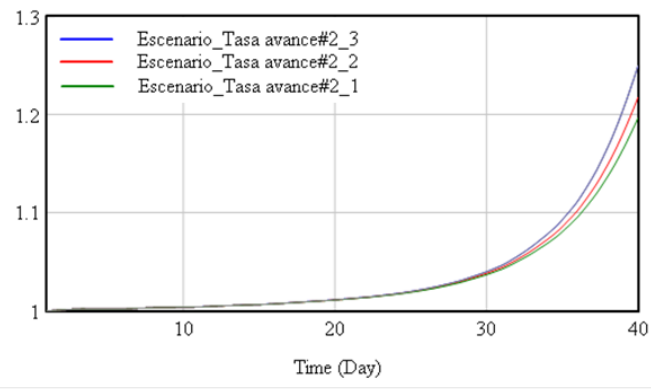

Estabilización Aprendizaje Grupos t

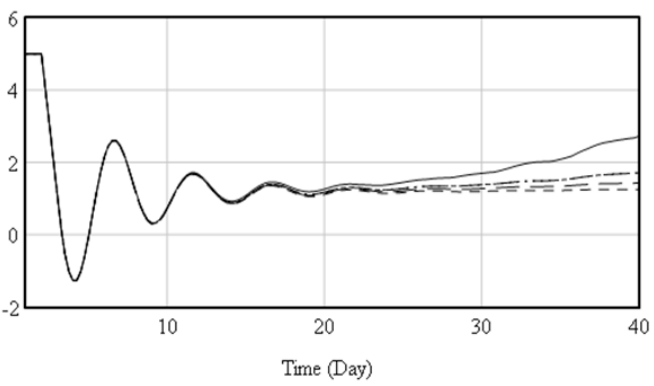

Nivel de aprendizaje Grupos t

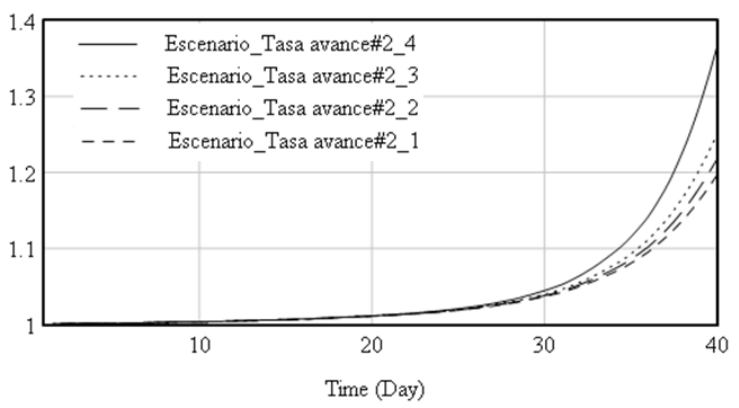

"Motivación-control Profesor"

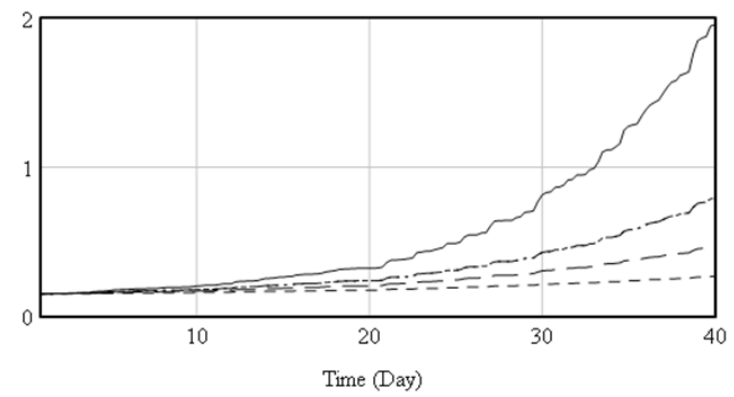


Sin embargo, EAG comienza a mostrar indicios de inestabilidad, que en el extremo podrían colapsar el proceso. Así mismo MCP requiere cambio en la misma dirección que NAG. Las simulaciones realizadas no tienen un impacto de forma inmediata significativo sobre NIG. La función logística de la figura 10 confirma el hecho de que el aprendizaje asociado a la investigación requiere tiempo y esfuerzo.
Figura 10 Simulación del Nivel de Investigación con Tasa avance \#2

Fuente: Elaboración propia
Nivel de Investigación Grupos t

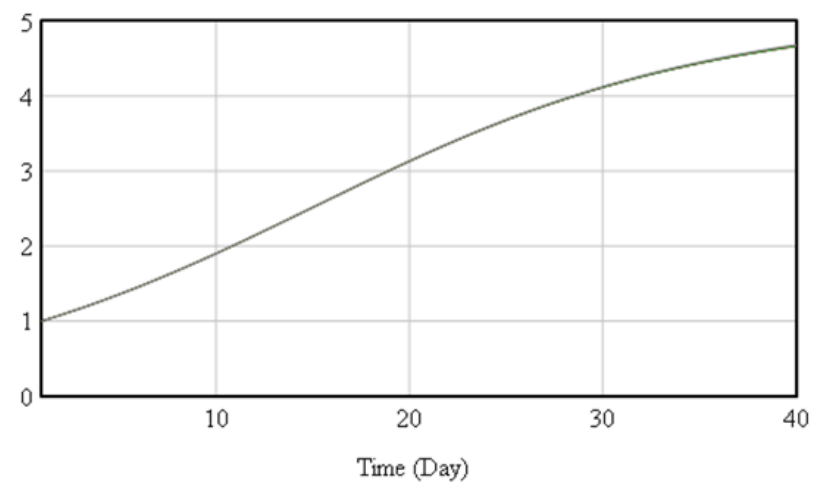

Siguiendo los mismos supuestos que con "Tasa avance \#2", en la figura 11, se muestran las simulaciones interviniendo en "Tasa avance \#1". En la gráfica de la parte izquierda, se muestran las evoluciones de niveles según tasas de avances. En los gráficos de la zona derecha, obtenidos del flujo, se puede observar un crecimiento de tipo exponencial en las primeras 20 clases. Los cambios de tendencia se observan a las $5^{\mathrm{a}}, 9^{\mathrm{a}}$ y $15^{\mathrm{a}}$ clase, como consecuencia del bucle retroalimentación positiva. Aproximadamente a la $15^{\mathrm{a}}$ clase línea de color verde en el caso del ritmo más lento de avance, la tendencia cambia la concavidad. Los grupos están empezando a sentir la contención del proceso de asimilación y desarrollo de conocimientos y habilidades, como consecuencia de la complejidad, o la disponibilidad de recursos. Volviendo a las trayectorias de la figura de la izquierda. El crecimiento exponencial se convierte en un crecimiento asintótico.
Figura 11 Simulación del Nivel de Investigación con Tasa de avance \#1 Fuente: Elaboración propia
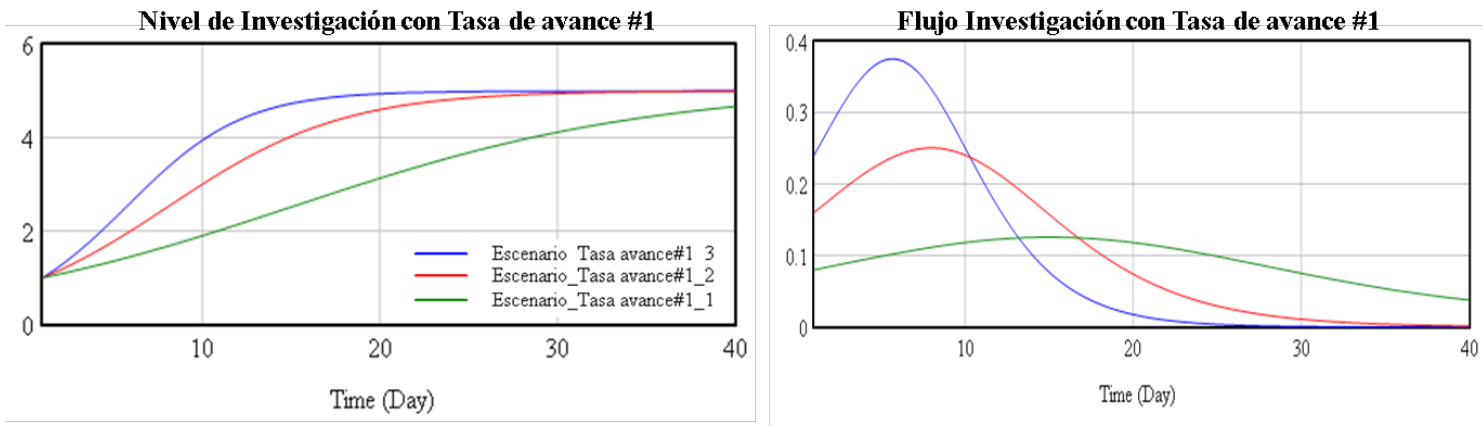

El punto de la curva en el cual cambia la concavidad es el punto de inflexión y está situado en el valor fijado en la “Tasa de grupos \#2" equivalente a 5 grupos. A medida que el valor anterior se incrementa la pendiente de la concavidad, también lo hace, hasta un límite aproximadamente de 16 grupos, donde el sistema se colapsaría.

Finalmente, en la figura 12, se muestra la evolución de tendencias de la motivación global de grupos, bajo la simulación de cambios en "Demora \#2" del 20\%. 
Figura 12 Simulación de la Motivación Global Grupos con Demora \#2

Fuente: Elaboración propia

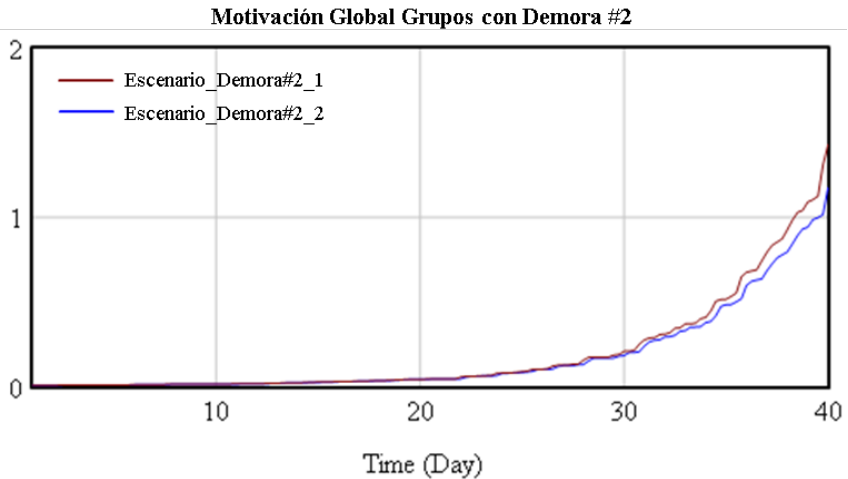

\section{Análisis de la evaluación de la asignatura}

En esta sección se aborda el análisis de la evaluación de DEE con el $88 \%$ del curso desarrollado. El proceso de recolección de la información se llevó a cabo a través de los coordinadores de cada grupo. Por tanto, aunque las encuestas son anonimizadas, y no es posible identificara1@s alumn@s encuestados, existe cierto sesgo al poder identificar al grupo. En la tabla 1, se muestran los resultados obtenidos en términos de sus valores medios. En términos generales, los valores medios son elevados en todas las preguntas. L@s alumn@s valoran positivamente, los recursos disponibles para poder seguir la asignatura durante el confinamiento (P1) con 8.5 puntos.
Tabla 1 Estadística

descriptiva de las preguntas realizadas

Fuente: Elaboración propia

\begin{tabular}{|c|c|c|c|c|c|c|}
\hline Id & Item & Media & $\mathrm{DE}$ & Min. & Max. & $\mathrm{n}^{\circ}$ \\
\hline P1 & $\begin{array}{l}\text { El profesor ha puesto a tu disposición los recursos telemáticos adecuados } \\
\text { para seguir el ritmo de las actividades, de la asignatura en la elaboración } \\
\text { del trabajo a desarrollar }\end{array}$ & 8.5 & 1.15 & 6 & 10 & 61 \\
\hline P2 & Cuál es tu grado de satisfacción con relación a: (cuestionario UAH) & & & & & \\
\hline 2.1 & Claridad de sus explicaciones & 7.0 & 1.26 & 4 & 10 & 61 \\
\hline 2.2 & Su disponibilidad a contestar las preguntas/dudas & 9.5 & 0.67 & 7 & 10 & 61 \\
\hline 2.3 & Su disponibilidad para las tutorías (on-line) & 8.5 & 1.39 & 5 & 10 & 58 \\
\hline 2.4 & Su corrección y respeto hacia los estudiantes & 9.4 & 1.19 & 5 & 10 & 61 \\
\hline 2.5 & Su capacidad para fomentar la participación & 7.7 & 1.14 & 4 & 10 & 55 \\
\hline P3 & Valora los siguientes recursos que has utilizado & & & & & \\
\hline 3.1 & dropbox: intercambio de archivos, información y evaluación & 8.9 & 1.17 & 5 & 10 & 49 \\
\hline 3.2 & email: intercambio de archivos, información y evaluación & 8.7 & 0.88 & 6 & 10 & 46 \\
\hline 3.3 & slack: mensajes escritos, video llamadas, intercambio de archivos & 8.0 & 1.52 & 4 & 10 & 49 \\
\hline 3.4 & skype & 8.2 & 1.23 & 6 & 10 & 29 \\
\hline 3.5 & jitsi & 8.7 & 1.1 & 4 & 10 & 45 \\
\hline P4 & $\begin{array}{l}\text { Durante el confinamiento y hasta la fecha de la encuesta, has tenido diferentes } \\
\text { controles del desarrollo del trabajo, recibiendo información concreta } \\
\text { (conceptos, metodologías, estructura del trabajo, etc) Valorar }\end{array}$ & & & & & \\
\hline 4.1 & Globalmente & 8.1 & 1.25 & 5 & 10 & 58 \\
\hline 4.2 & Control 18_03_2020 & 7.9 & 1.24 & 5 & 10 & 61 \\
\hline 4.3 & Control 15_04_2020 & 8.1 & 1.29 & 5 & 10 & 61 \\
\hline 4.4 & Control 25_04_2020 & 8.0 & 1.16 & 6 & 10 & 61 \\
\hline 5 & Como valoras el estado actual de desarrollo de tu trabajo & 7.7 & 0.94 & 6 & 10 & 60 \\
\hline
\end{tabular}

En particular, la utilización de dropbox, correo electrónico, o la plataforma JITSI con 8.9;8.7;8.7 respectivamente. Las preguntas con valores menores son; la propia valoración del desarrollo de su trabajo; capacidad para fomentar la participación y la claridad de las explicaciones con 7.7; 7.7; $7 ; 0$ respectivamente.
El análisis Clúster (método de Ward) ha permitido segmentar la muestra en dos grupos (grupo \#1=17 alumn@s $32 \%$ y grupo \#2=36 alumn@s 68\%) a partir de las preguntas realizadas. La tabla 2, muestra las diferentes evaluaciones según grupos, más elevadas en el caso del grupo \#2. 
Tabla 2 Estadística descriptiva de las preguntas realizadas según grupos Fuente: Elaboración propia

\begin{tabular}{|c|c|c|c|c|}
\hline Id & Item & Grupo \#1* & Grupo \#2* & F(Pvalor) \\
\hline 1 & $\begin{array}{l}\text { El profesor ha puesto a tu disposición los recursos telemáticos adecuados } \\
\text { para seguir el ritmo de las actividades, de la asignatura en la elaboración } \\
\text { del trabajo a desarrollar }\end{array}$ & $7.88(1.45)$ & $8.56(0.93)$ & $432.0(0.042)$ \\
\hline 2.1 & Claridad de sus explicaciones & $5.64(0.78)$ & $7.56(1.03)$ & $45.86(0.000)$ \\
\hline 2.2 & Su disponibilidad a contestar las preguntas/dudas & $8.82(0.72)$ & $9.80(0.41)$ & $40.25(0.000)$ \\
\hline 2.3 & Su disponibilidad para las tutorías (on-line) & $7.23(1.25)$ & $9.05(1.11)$ & $28.30(0.000)$ \\
\hline 2.4 & Su corrección y respeto hacia los estudiantes & $8.38(1.55)$ & $9.77(0.72)$ & $20.12(0.000)$ \\
\hline 2.5 & Su capacidad para fomentar la participación & $7.17(1.38)$ & 7.95(0.95) & $5.75(0.0200)$ \\
\hline 4 & $\begin{array}{l}\text { Durante el confinamiento y hasta la fecha de la encuesta, has tenido } \\
\text { controles del desarrollo del trabajo, recibiendo información concreta } \\
\text { (conceptos, metodologías, estructura del trabajo, etc) Valorar }\end{array}$ & $7.14(1.27)$ & $8.51(1.06)$ & $16.76(0.000)$ \\
\hline 5 & Como valoras el estado actual de desarrollo de tu trabajo & $7.61(1.30)$ & $7.71(0.72)$ & $0.140(0.708)$ \\
\hline
\end{tabular}

*Media(Desv. Est.)

El análisis discriminante confirma una clasificación al 98.11\% (Lambda de Wilks=0.240; chi2=67.05; Pvalor=0.00). Se pidió a los alumn@s que realizarán los comentarios que considerarán oportunos relativos al proceso enseñanzaaprendizaje de DEE. Posteriormente se realizó un análisis cuantitativo, basado en estos, por medio de la herramienta nube de palabras y análisis de red. En relación con el primer análisis, la figura 13, muestra la relevancia que los alumn@s otorgan a "trabajo"; "asignatura", "profesor", "proceso", etc.
Figura 13 Nube de

palabras en relación con

los comentarios de 1@s

estudiantes

Fuente: Elaboración propia

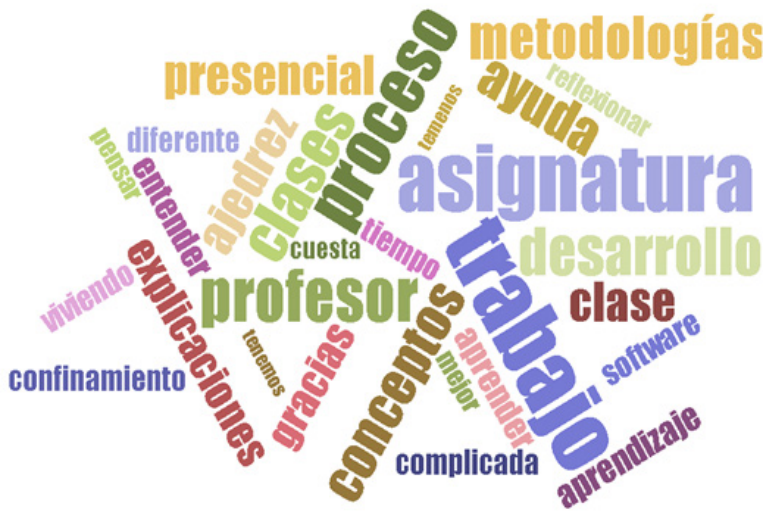

A partir de una matriz de adyacencia elaborada a través de los comentarios realizados por los estudiantes y las veces que estos coinciden en ellos, la figura 14, recoge un análisis de red. 
Figura 14 Análisis de red en relación con los comentarios de 1@s estudiantes

Fuente: Elaboración propia

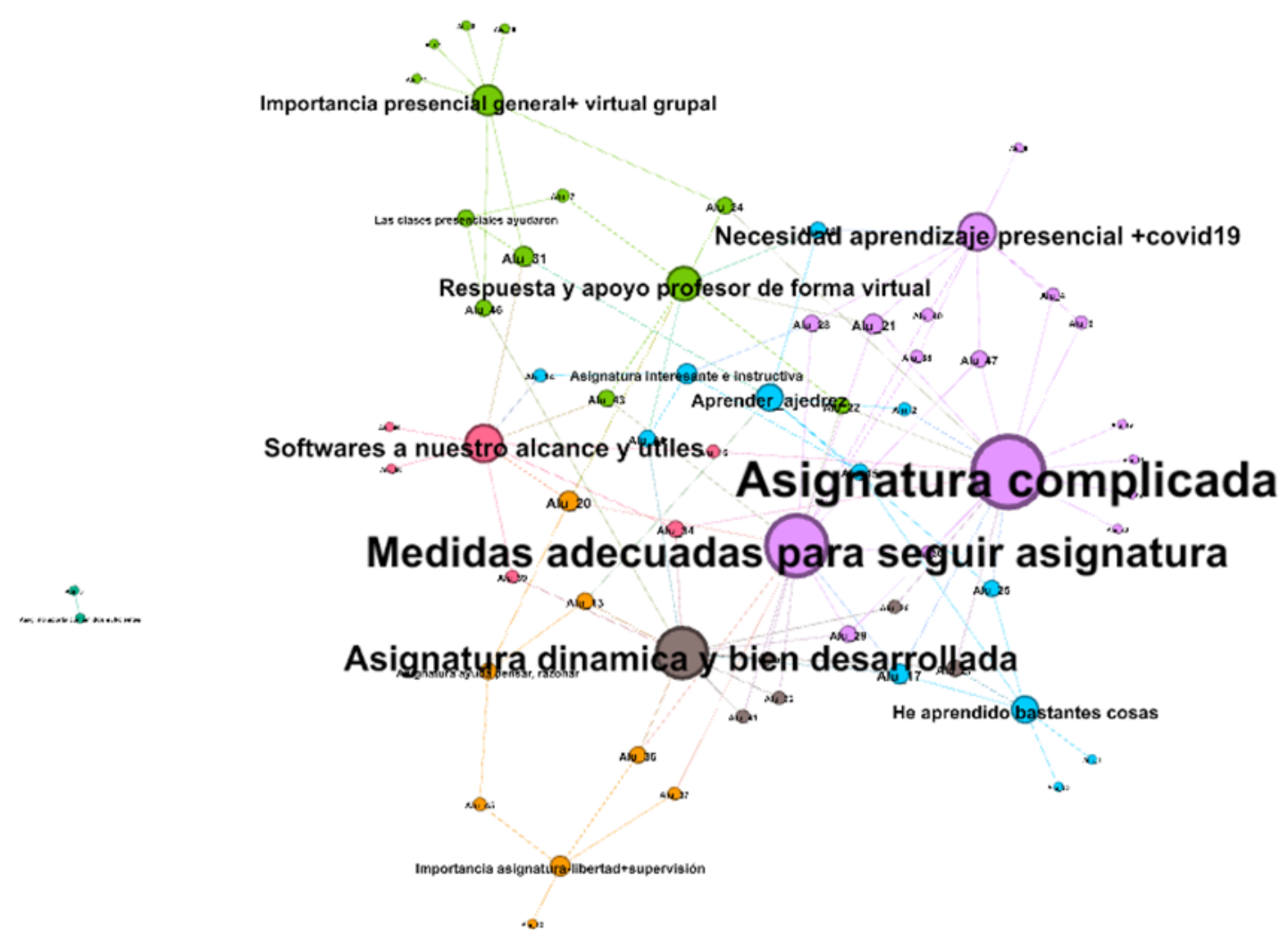

El análisis fue realizado con el software gephi. Se aplico el algoritmo force atlas y posteriormente se utilizó la tipología por grado y modularidad. La formación de los nodos y comunidades muestra la representatividad de los alumnos y la pertenencia a las diferentes comunidades que por ejemplo consideran la "asignatura complicada" asociados con las "medidas adecuadas para seguir la asignatura" y la importancia del "aprendizaje presencial por la covid-19". Otra comunidad, está formada por alumnos que se relacionan con que la asignatura es dinámica y bien desarrollada entre otras.

\section{Conclusiones}

Este trabajo, se ha planteado como objetivo documentar el proceso de enseñanza-aprendizaje, en la asignatura de Dirección Estratégica Empresarial de $4^{\circ} \mathrm{ADE}$ en el curso 2020, a partir de la elaboración de un trabajo de investigación, por medio de un proceso pedagógico, donde el aprendizaje es construido, autónomo y colaborativo. Para ello se ha utilizado la metodología de sistemas dinámicos. A partir de la hipótesis dinámica y por tanto del establecimiento de las relaciones causales, que asocian motivación (desde sus diferentes perspectivas) de los alumnos/grupos y del docente con el proceso de aprendizaje. Los principales resultados alcanzados, por medio de la simulación y de los escenarios planteados han sido los siguientes. El modelo planteado refleja el comportamiento del proceso de enseñanza y su validación se ajusta razonablemente a la información y percepción del proceso.

Las suposiciones en condiciones extremas proporcionaron respuestas relativamente razonables. Por ejemplo;

1) cambios experimentados en propuestas de la tasa de avance de los trabajos (10\% a 30\%) en la concreción de los objetivos o/y las metodologías más idóneas o/y el tipo de información necesaria, desde la motivación y control del docente, influyen de forma exponencial en mayores niveles de exigencia de este. En estas circunstancias la estabilización del proceso se establece apropiadamente en la mediana del número de clases y tutorías y se ve su influencia en el proceso de aprendizaje de los alumnos, de forma exponencial. Cuando se proponen avances que alcanzan el nivel del $50 \%$, el aprendizaje tiende a evolucionar, pero comienzan a aparecer señales de inestabilidad.

2) ante propuestas de cambios con aumentos en el retardo de la estabilización (relación entre la heterogeneidad de conocimientos y el tipo de tutoría asistencial o integral que genera problemas de ajuste de atención a los grupos) en Demora \#1, el sistema se vuelve inestable, dado que la descoordinación aumenta.

3) la evolución en forma logística del conocimiento en investigación implica que evoluciones en aprendizaje no se relacionan instantáneamente con aquellos, pero cambios 
en la tasa de aprendizaje (10\% a $30 \%$ sobre 5 a 10 grupos) relacionados exclusivamente al nivel de investigación provocan mejoras de acuerdo con el patrón logístico. Cuando se modifica la tasa de grupos \#2 a niveles superiores a 14 grupos el sistema colapsa.

La experiencia docente documentada a través de la dinámica de sistemas podría servir como laboratorio de simulación cualitativo de soporte para la experimentación controlada, donde los efectos de los escenarios pueden ser contrastados, proporcionando la posibilidad de recomendaciones y mejoras de futuro. Sin dejar a un lado la importancia de las clases magistrales, es probable, que compaginar estas con procesos de enseñanza-aprendizaje más dinámicos y proactivos para el alumno pueda ser un camino de mejora. Esta vía de actuación no está exenta de obstáculos. Por un lado, es necesario, un mayor compromiso y dedicación del docente. El paso de una docencia centrada en el profesor a otra más vinculada al alumno y la metodología, donde aquel interactúa en forma de catalizador del proceso, es complejo. Por el momento, la ayuda del docente es su motivación intrínseca, dado que el sistema universitario, no parece tener mecanismos explícitos de reconocimiento de buenas prácticas docentes. Además, es importante considerar, la fuerte vinculación hacia actividades de investigación, más fáciles de evaluar. Por otro lado, se requiere un comportamiento proactivo por parte de los alumnos, atendiendo a la heterogeneidad en conocimientos, actitudes y aptitudes existentes en el aula. Se trata de salvar obstáculos que requieren fuertes dosis de compromiso, pero que a su vez suponen un reto motivador, en los tiempos actuales, de enorme complejidad e incertidumbre.

Extensiones de este trabajo podrían ir encaminadas a estudiar en mayor profundidad la relación entre el nivel de aprendizaje y el de investigación a través de variables intermedias y conservando la potencia holística de la metodología utilizada. Tambien podría ser interesante, el concepto de virtualización del proceso al 100\%.

\section{Agradecimientos}

El autor, de este trabajo agradece la participación de 1@s alumn@s y su consentimiento para publicar los resultados de este, realizado entre enero-mayo 2020.

\section{Bibliografía}

ANDERSSON, C., KARLSSON, L., (2001). A system dynamics simulation study of a software development process. Master's thesis, Lund Institute of Technology, Lund University.
BARLAS, Y. (1996). Formal aspects of model validity and validation in system dynamics. System Dynamics Review. 12 (3); 183-210.

FORRESTER, J.W. (1968): Industrial dynamics. The MIT Press. Cambridge, Massachusetts. Traducción (1972): Dinámica industrial. Ed. El Ateneo. Buenos Aires.

HOMER, J. (2019). Best practices in system dynamics modeling, revisited: a practitioner's view. System Dynamics Review 35, (2) (April/June 2019): 177-181

LOCKE, E AND SCHATTKE, K. (2019). Intrinsic and extrinsic motivation: Time for expansion and clarification. Motivation Science, 5(4), 277-290.

PEREZ-ANSEDE, E. (2015). Revisión dinámica del modelo del multiplicador-acelerador de Samuelson. Universidad da Coruña. http://hdl.handle.net/2183/14607

ROBBINS, S.P. (2004), Comportamiento Organizacional. Pearson Educación.

STERMAN, J.D. (2000). Business Dynamics. McGrawHill, Inc. New York, NY, USA.

SUKHWAL, V. Monitoring students' growth using system dynamics. Graduate Theses and Dissertations. 14872. https://lib.dr.iastate.edu/etd/14872. Iowa State University

DE JORGE-MORENO, J. (2020). Apuntes de la asignatura de Dirección Estratégica Empresarial. http://www3. uah.es/justo_de_jorge/Docencia/docencia.htm/

FINKEL, D.L. (2008) Dar clase con la boca cerrada. Valencia: Servicio de Publicaciones Universidad de Valencia.

STERMAN, J.D. (2000). Business Dynamics: System thinking and modeling for a complex world. IrwinMcGraw-Hill.

WEBER, L. (2010). Demographic change and economic growth. Heidelberg: Physica Verlag.

YEPES, R. (2016). Arquetipos de sistemas: un conjunto de herramientas de conocimiento para explorar El valle de la muerte de la I+D. XIV Encuentro Colombiano de Dinámica de Sistemas / Medellín. 182 de 209 


\section{Anexo 1: Breve resumen de algunos de los trabajos}

\begin{tabular}{|c|c|}
\hline Objetivos/información & Datos y Metodología \\
\hline $\begin{array}{l}\text {-Análisis de la eficiencia de las Universidades Públicas Españolas } \\
\text { 2008/09-2017/18. } \\
\text {-Utilizan tres fronteras de referencia, dividiendo la muestra en función } \\
\text { del grado de especialización. }\end{array}$ & $\begin{array}{l}\text { Ministerio de Educación-CRUE } \\
\text { DEA intertemporal }\end{array}$ \\
\hline -Análisis competitivo del sector hotelero 2014-2018. & $\begin{array}{l}\text { SABI y diversas fuentes de información, como las } \\
\text { memorias, informes sectoriales. DEA, Análisis de red }\end{array}$ \\
\hline Análisis competitivo del sector del automóvil. El caso Toyota & $\begin{array}{l}\text { Cuentas anuales 2001-2019 } \\
\text { Revisión de la literatura. } \\
\text { Estadística descriptiva, DEA } \\
\text { Análisis de red y wordcloud }\end{array}$ \\
\hline $\begin{array}{l}\text { Un análisis del sector de fabricación de aviones. El caso del duopolio } \\
\text { Airbus-Boeing }\end{array}$ & $\begin{array}{l}\text { Cuentas anuales 1998-2018 } \\
\text { Revisión de la literatura. } \\
\text { Análisis de funciones de producción y costes por } \\
\text { métodos paramétricos y no paramétricos. } \\
\text { Teoría de Juegos }\end{array}$ \\
\hline Análisis de la eficiencia del sector minorista en España 1999-2018 & SABI -DEA + segunda etapa \\
\hline $\begin{array}{l}\text { Impacto de la gestión estratégica publica en la violencia de género en } \\
\text { España 2003-2020 }\end{array}$ & $\begin{array}{l}\text { Ministerio y BOE. } \\
\text { Análisis econometrico con regresiones de Poisson }\end{array}$ \\
\hline $\begin{array}{l}\text { Análisis de las audiencias de las principales cadenas de TV y la influencia } \\
\text { de los cambios directivos. 2006-2019 }\end{array}$ & $\begin{array}{l}\text { Barlovento, Informes del sector. } \\
\text { Series temporales y su predicción con modelos de } \\
\text { intervención }\end{array}$ \\
\hline Análisis de la eficiencia del sector bancario español 2005-2019 & $\begin{array}{l}\text { Información cuentas anuales } \\
\text { DEA intertemporal, Windows }\end{array}$ \\
\hline $\begin{array}{l}\text { Benchmark del sistema Universitario público en España. 2008/09- } \\
2017 / 18 \text {. }\end{array}$ & $\begin{array}{l}\text { Ministerio de Educación-CRUE } \\
\text { Análisis Multicriterio TOPSIS, WASPAS (entropía) }\end{array}$ \\
\hline
\end{tabular}

\section{Anexo 2: Recursos}

Se ha utilizado dropbox como herramienta flexible, para almacenar los trabajos y cualquier documento o archivo necesario, como plantillas en Excel, archivos del programa
Statgraphics Centurión XXI, Deap, Stata gretl, Yed o Stata. La tabla I, muestra los recursos de forma más concreta.

Tabla 1 Recursos utilizados

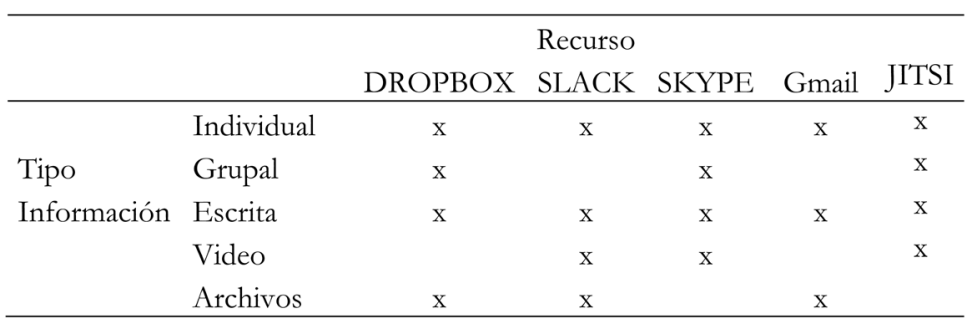

En la Tabla II, se muestran las fases y el cronograma con las actividades. La fase 1, comprende la formación de grupos y los intentos de concretar la idea del trabajo. Este supone el $100 \%$ de la nota, en caso de superar el nivel exigido de calidad. Normalmente se produce una tutorización presencial a lo largo del semestre con dos controles al 50\% y $75 \%$ de las clases. En este último control se le indicará al alumno si debe o no presentarse a un examen final. La evaluación del trabajo es del $80 \%$ por parte del profesor y $20 \%$ coevaluación ( $10 \%$ intragrupos $+10 \%$ intergrupos). Sin embargo, las condiciones de confinamiento por el covid-19, han supuesto cinco controles 
Tabla 2 Fases, Cronograma $\mathrm{y}$ actividades

\begin{tabular}{|c|c|c|c|}
\hline Fase & Cronograma & Actividad & Observaciones \\
\hline 1. Formación del grupo & $1^{\mathrm{a}}$ a $4^{\mathrm{a}}$ semana & $\begin{array}{l}\text { Formación de los } \\
\text { integrantes del grupo }\end{array}$ & $\begin{array}{l}\text { Realizarán el trabajo global de } \\
\text { la asignatura }\end{array}$ \\
\hline $\begin{array}{l}\text { 2. Clases magistrales } \\
\text { teóricas y prácticas }\end{array}$ & $1^{\underline{a}}$ a $10^{\underline{a}}$ semana & $\begin{array}{l}\text { Explicación de } \\
\text { conceptos y de } \\
\text { metodologías. Los } \\
\text { alumnos utilizan sus } \\
\text { ordenadores en el aula }\end{array}$ & $\begin{array}{l}\text { Todo el material está disponible } \\
\text { en dropbox antes de las clases. } \\
\text { Trasparencias, papers, } \\
\text { programas. }\end{array}$ \\
\hline $\begin{array}{l}\text { 3. Trabajo grupal en el } \\
\text { aula. Se introducen } \\
\text { mínimas clases } \\
\text { magistrales } 10 \text { a } 15 \\
\text { min. }\end{array}$ & $11^{\mathrm{a}}$ a $19^{\mathrm{a}}$ semana & $\begin{array}{l}\text { Desarrollo de } \\
\text { actividades } \\
\text { específicamente } \\
\text { vinculadas a los } \\
\text { trabajos }\end{array}$ & $\begin{array}{l}\text { Dentro de las actividades a } \\
\text { desarrollar, se contemplan, } \\
\text { conceptos o metodologías, a } \\
\text { nivel, grupal, que no han sido } \\
\text { consideradas a nivel global. }\end{array}$ \\
\hline $\begin{array}{l}\text { 4. Exposición de } \\
\text { trabajos para su } \\
\text { evaluación }\end{array}$ & $\begin{array}{l}\text { Fecha limite día } \\
\text { anterior al examen }\end{array}$ & $\begin{array}{l}\text { Entrega y mejora del } \\
\text { trabajo }\end{array}$ & $\begin{array}{l}\text { Debido al covid-19, exposición } \\
\text { de todos los trabajos en una } \\
\text { carpeta de dropbox. }\end{array}$ \\
\hline
\end{tabular}

\section{Anexo 3: Formulación}

\begin{tabular}{|c|c|c|c|}
\hline $\begin{array}{c}\text { Nombre de la } \\
\text { variable }\end{array}$ & $\begin{array}{l}\text { Valor } \\
\text { inicial }\end{array}$ & Ecuación/rango & Unidad \\
\hline $\begin{array}{l}\text { Motivación global } \\
\text { grupos t } \\
\text { (MGG) }\end{array}$ & 0.02 & $\int_{0}^{t}($ Flujo $M G G * N I G) d \emptyset+V I(M G G)$ & Dmnl \\
\hline $\begin{array}{l}\text { Nivel Aprendizaje } \\
\text { Grupos t } \\
\text { (NAG) }\end{array}$ & 1 & $\int_{0}^{t}($ Flujo $N A G) d \emptyset+V I(N A G)$ & Dmnl \\
\hline $\begin{array}{l}\text { Nivel de } \\
\text { Investigación } \\
\text { grupos t (NIG) }\end{array}$ & 1 & $\int_{0}^{t}($ Flujo $N I G) d \emptyset+V I(N I G)$ & Dmnl \\
\hline $\begin{array}{l}\text { Estabilización } \\
\text { Aprendizaje } \\
\text { grupos t } \\
\text { (EAG) }\end{array}$ & 5 & $\int_{0}^{t}($ Flujo FEA*NAG)dØ+VI(EAG) & Dmnl \\
\hline $\begin{array}{l}\text { Conocimientos } \\
\text { Experiencia } \\
\text { Profesor } \\
(\mathrm{CEP})\end{array}$ & 0.15 & $\int_{0}^{t}($ Flujo $F R * N A G * N I G) d \emptyset+V I(C E P)$ & Dmnl \\
\hline $\begin{array}{l}\text { Ruido efectos } \\
\text { exógenos } \\
\text { motivación } \\
\text { (RFM) }\end{array}$ & & $\begin{array}{l}\text { SMOOTH (RANDOM NORMAL (Mínimo, } \\
\text { Máximo, Media, Desv. Estándar, semilla, 1) 1.25- } \\
\text { 0.9-1-0.5-1234 }\end{array}$ & Dmnl \\
\hline $\begin{array}{l}\text { Motivación } \\
\text { Extrínseca (ME) }\end{array}$ & & $\begin{array}{l}\text { RANDOM UNIFORM (Media, Desv. Estándar, } \\
\text { semilla) }(0.05,0.7,123456)\end{array}$ & Dmnl \\
\hline $\begin{array}{l}\text { Motivación } \\
\text { Intrínseca (MI) }\end{array}$ & & $\begin{array}{l}\text { RANDOM UNIFORM (Media, Desv. Estándar, } \\
\text { semilla) }(0,0.5,123456)\end{array}$ & Dmnl \\
\hline $\begin{array}{l}\text { Motivación } \\
\text { Necesidad de } \\
\text { logro (MNL) }\end{array}$ & & $\begin{array}{l}\text { RANDOM UNIFORM (Media, Desv. Estándar, } \\
\text { semilla) }(0,1,123456)\end{array}$ & Dmnl \\
\hline Brecha \#1 & & (Tasa \#1-CEP) / Tasa \#1 & Dmnl \\
\hline Tasa \#1 & & 5 & grupos \\
\hline $\begin{array}{l}\text { Tasa } \\
\text { avance \#2 }\end{array}$ & & 0.2 & Dmnl \\
\hline $\begin{array}{l}\text { Tasa } \\
\text { grupos \#1 }\end{array}$ & & 2 & grupos \\
\hline Demora \#1 & & 1 & Dmnl \\
\hline $\begin{array}{l}\text { Tasa } \\
\text { Aprendizaje \#1 }\end{array}$ & & 0.02 & Dmnl \\
\hline $\begin{array}{l}\text { Tasa } \\
\text { grupos \#2 }\end{array}$ & & 5 & Dmnl \\
\hline Brecha \#2 & & $($ Tasa \#2-CEP) / Tasa \#2 & Dmnl \\
\hline Tasa avance \#1 & & 0.2 & Dmnl \\
\hline Demora \#2 & & 1 & Dmnl \\
\hline
\end{tabular}

Dmnl=Unidad de medida adimensional, empleada en los sistemas complejos (epidemiología, educación etc.) 